\title{
Light and scanning electron microscopy of the tongue of the sand lizard (Lacerta agilis)
}

\author{
P. Cizek', P. Hamouzova1', P. Kvapil², M. Kyllar¹, 3 \\ ${ }^{1}$ Department of Anatomy, Histology and Embryology, Faculty of Veterinary Medicine, \\ University of Veterinary and Pharmaceutical Sciences Brno, Czech Republic \\ 2ZOO Ljubljana, Slovenia \\ ${ }^{3}$ Companion Care Vets, United Kingdom
}

[Received: 11 May 2018; Accepted: 8 June 2018]

Background: Despite the fact that numerous reptile species are widely studied by the researchers, information describing the detailed structure of particular organs in many reptiles is missing.

Materials and methods: The tongue of the sand lizard (Lacerta agilis) was examined under the light and scanning electron microscope. It is divided into bifurcated apex, corpus and bifurcated radix. The tip of the lingual apex is devoid of lingual papillae. Results: The remaining dorsal surface of the tongue bears either fused papillae in the form of caudally directed ridges or individual papillae represented by mushroom-like or semilunar prominences (lingual apex) or fish scale-like papillae (lingual corpus) and horizontally laid ridges extending in the form of lobulated prominences (lingual corpus, lingual radix). Regardless of the shape, lingual papillae contain numerous muscle fibres and they are all considered to be mechanical. The lingual epithelium changes from the simple squamous into stratified squamous in the caudal direction. No salivary glands or sensory structures were recognised. Conclusions: This description is to be used mainly for comparative studies. It could also help to understand how different lizards capture the pray. (Folia Morphol 2019; 78, 1: 101-106)

Key words: lingual papillae, Lacertidae, morphology, reptiles, scanning electron microscope

\section{INTRODUCTION}

The tongue is a muscular organ located in the oral cavity. The type of food intake and processing of food in animals are important factors that affect the morphological diversity of structures on the surface of the tongue. The tongue plays a prominent role in the intra-oral transport and deglutition of food. This important role has led to morphological studies of several animals' tongues to examine differences and similarities among them in terms of feeding habits and also to identify relations among diverse species [4].
Lacerta agilis is a small to medium sized lizard, strongly diurnal, largely insectivorous, actively chasing and consuming a range of spiders and insects, especially orthopterans, bugs and beetles. This species is also known to eat its own young [7].

The morphology of the tongue has been described in various species of turtles [12, 14, 15, 19]. In lizards, the lingual morphology is still unknown in many species. The lack of detailed description in specific species has hindered the understanding of the functional morphology of the lizard tongue, very important for the description of lingual pathologies. Therefore the aim

Address for correspondence: Dr. P. Cizek, Department of Anatomy, Histology and Embryology, Faculty of Veterinary Medicine, University of Veterinary and Pharmaceutical Sciences Brno, Palackeho 1946/1, 61242 Brno, Czech Republic, tel: +420 737729863, e-mail: cizekpetr@yahoo.com 
of the present study was to describe the morphology of the tongue of the sand lizard by light and scanning electron microscopy. The results are helpful to better understand the food processing and also the medical approach in the oral cavity disorders in lizards.

The tongue of squamates is connected to the floor of the oral cavity and resembles the flat and less keratinised tongue of some amphibians. Some lizards, as well as turtles, cannot protrude their tongue. Despite this fact, the lingual apex of many lizards is forked and the lingual radix also extends distally in the form of two processes [6]. However, the tongue of the rough tailed rock agama (Laudakia stellio) is not forked [16].

The dorsal surface of the tip of the tongue is smooth in many lizards such as the green anole, Anolis carolinensis [18], blue-tongued skink, Tiliqua scincoides [2], Japanese lizard, Takydromus tachydromoides [13], bearded dragon, Pogona vitticeps [21], Italian lizard, Podarcis sicula [3], rough tailed rock agama, Laudakia stellio [16] and green iguana, Iguana iguana [1, 6].

Cylindriform papillae were distinguished on the dorsal lingual surface in the green anole, Anolis carolinensis [18], blue-tongued skink, Tiliqua scincoides [2], rough tailed rock agama, Laudakia stellio [16], green iguana, Iguana iguana [1, 6], Madagascan collared iguana, Oplurus cuvieri [8], Italian lizard, Podarcis sicula [3] and bearded dragon, Pogona vitticeps [21]. Plumose papillae were described in the green anole, Anolis carolinensis [18] and bearded dragon, Pogona vitticeps [21]. The plumose papillae may be important in mastication and deglutition of food [18]. Foliate-like papillae were found in the Italian lizard, Podarcis sicula [3] and blue-tongued skink, Tiliqua scincoides [2], conical papillae were described in the Madagascan collared iguana, Oplurus cuvieri [8], green iguana, Iguana iguana [1], conical flattened filiform papillae in the ringed wall gecko, Tarentola annularis [9], fungiform papillae in the rough tailed rock agama, Laudakia stellio [16] and scale-like papillae in the Japanese lizard, Takydromus tachydromoides [13], Tsinling dwarf skink, Scincella tsinlingensis [20] and Gekko japonicus [11]. Regardless of type, all lingual papillae of the green anole (Anolis carolinensis) contain a single longitudinally oriented skeletal muscle fibre that originates from the underlying lingual muscles [18].

\section{MATERIALS AND METHODS}

\section{Animals}

Three adult males and one female sand lizard (Lacerta agilis) used in this study were born and kept in a zoological collection. Their age varied between 4 and 6 years. The animals were fed with a balanced diet that consisted mostly of insects and vitamin-mineral supplements. All the animals died or were euthanized due to various reasons related to old age (internal organ failure, neoplasia etc.) at the veterinary ambulance of Ljubljana ZOO. None of the animals used in this study suffered from any of the oral cavity disorders.

\section{Light microscopy}

The tongues were fixed in $4 \%$ neutral buffered formaldehyde for 5-7 days. After fixation had been completed, samples for light microscopy were dehydrated in a graded alcohol series (the ethanol concentration in each subsequent bath was increased by $10 \%)$, acetone, and three baths of xylene. At the end of dehydration process, samples were infiltrated with hot paraffin and embedded in paraffin wax. Then, 3-4 $\mu \mathrm{m}$ thin sections were cut in the routine manner. The sections were dried, stained with haematoxylin and eosin, mounted and examined, and finally photographed under an Olympus BX51 light microscope using an Olympus DP70 digital camera.

\section{Scanning electron microscopy}

The samples for scanning electron microscopy were dehydrated in a graded alcohol series $(60,70$, 96 and $100 \%, 20$ min for each concentration), transferred to pure acetone, dried at the critical point (Baltec CPD 030 Critical Point Dryer, Bal-Tec, UK), coated with gold (Balzers SCD 040 using a current $30 \mathrm{~mA}$ for $4 \mathrm{~min}$ ) and finally examined and photographed under a Tescan VEGA TS 5136 XM scanning electron microscope in a high vacuum and accelerated voltage $20 \mathrm{kV}$ by using an SE detector.

\section{RESULTS}

The tongue of the sand lizard (Fig. 1) is dorsoventrally flattened and roughly wedge shaped. It is divided into three areas - bifurcated apex, corpus and bifurcated radix. The tip of the lingual apex (Fig. 2) is relatively smooth and devoid of papillae. Approximately in the middle of the bifurcated apex, caudally directed ridges are formed. These ridges then separate into individual papillae (Fig. 3). Each papilla is represented by a mushroom like prominence and measures around $100 \mu \mathrm{m}$ in diameter. Papillae covering the margin of the apex of the tongue are different. On its lateral surface, these papillae tend to be bigger. Medially they appear as a few semilunar 


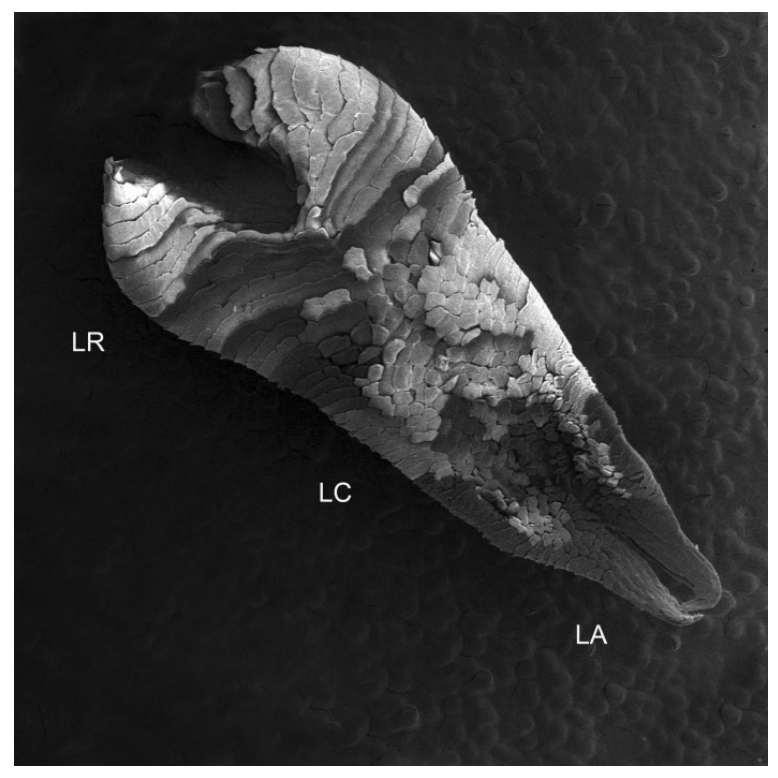

Figure 1. Tongue of a sand lizard; LA — lingual apex; LC — lingual corpus; LR - lingual radix.

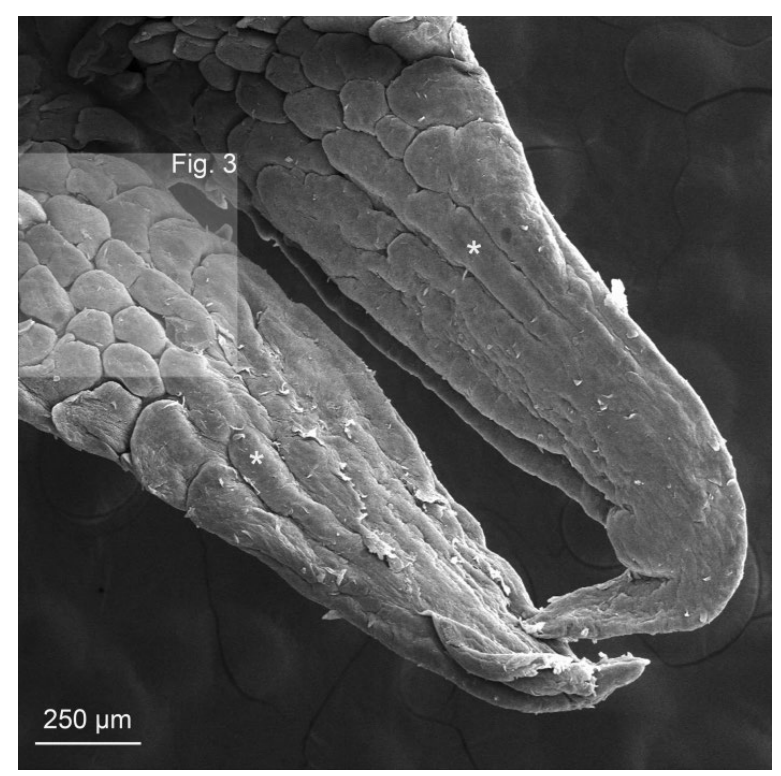

Figure 2. Lingual apex. Asterisks indicate caudally directed ridges.

prominences. Regardless the shapes of the papillae, the interpapillary grooves are rather inconspicuous.

The papillae in the rostral part of the lingual body (Fig. 4) are rostrocaudally flattened. Each papilla bends caudally and extends in the form of several lobulated prominences. Their shape resembles a fish scale. Papillae covering the lingual body are roughly twice as big compared to the papillae on the apex, i.e. around $200 \mu \mathrm{m}$. On the margin of the lingual body, the papillae are even more flattened and their appearance changes into horizontally laid, long, narrow

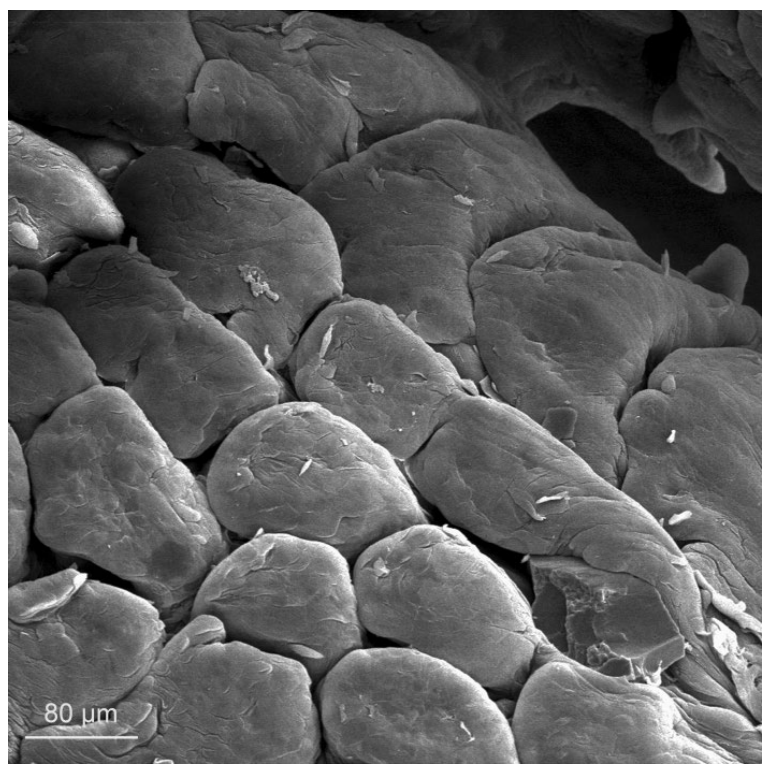

Figure 3. Lingual apex. Detail. Ridges separate into individual papillae.

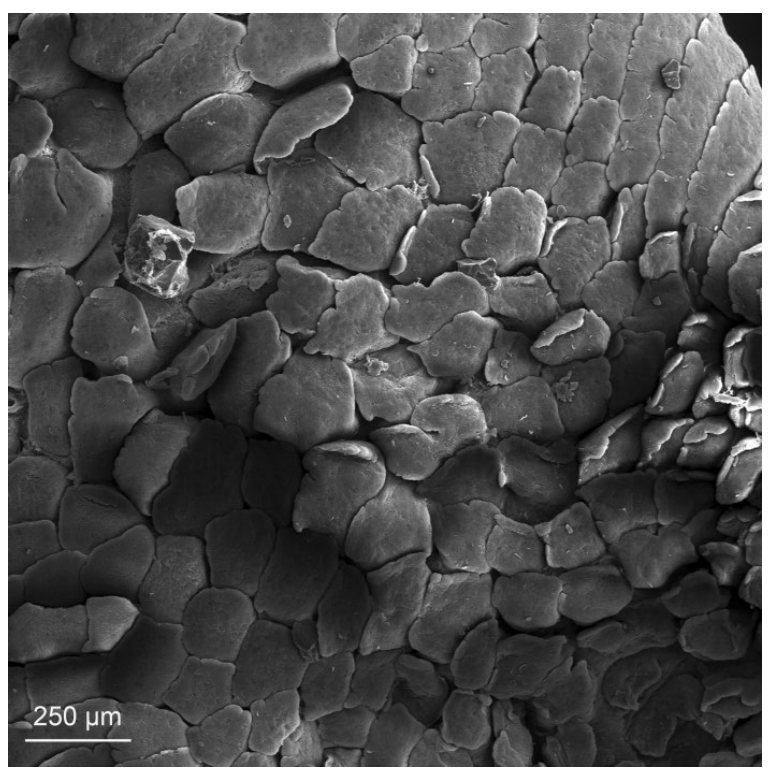

Figure 4. Lingual corpus. Caudally bent papillae on its rostral portion.

prominences that remarkably resemble a few merged papillae (Fig. 5). These papillary ridges extend in the form of similar lobulated prominences as the papillae in the medial area of the lingual body. Some of these lobes are separated by clearly evident notches. In the caudal direction medially (Fig. 6), papillary ridges elongate so they cover the entire caudodorsal lingual surface of the lingual body. Subsequently, individual scale-like papillae gradually disappear.

The lingual radix (Fig. 7) is bifurcated as well as the lingual apex. Its margin is more rounded compared 


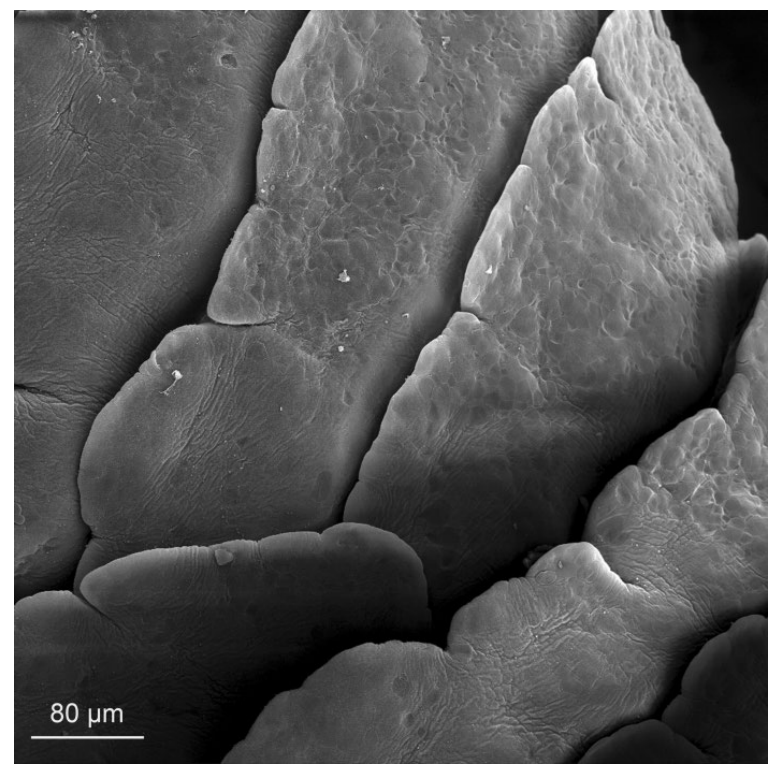

Figure 5. Lingual corpus. Papillae on its margin.

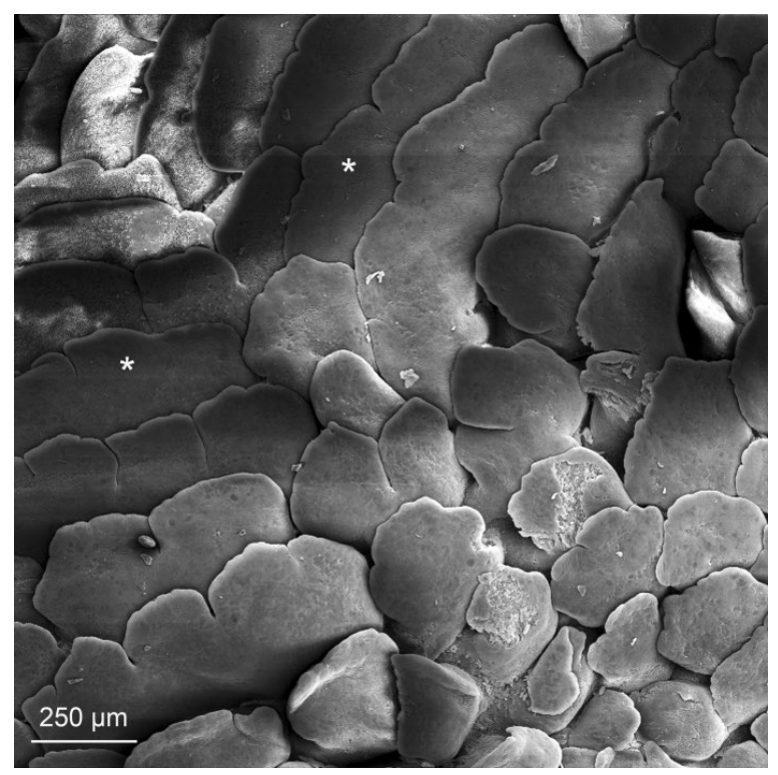

Figure 6. Lingual corpus. Papillary ridges on its distal portion.

to the proximal portions of the tongue. The dorsal lingual surface bears the same papillary ridges that are found on the lingual body (Fig. 8). The interpapillary notches are very deep.

The superficial cells covering all the various papillae of the dorsal lingual surface are densely covered with microvilli.

A simple squamous epithelium covers the lingual papillae on the apex. Aborally, the number of layers of epithelial cells increases. Nonkeratinised stratified squamous epithelium is on the surface of papillae on the body and radix. Papillary cores are formed by the

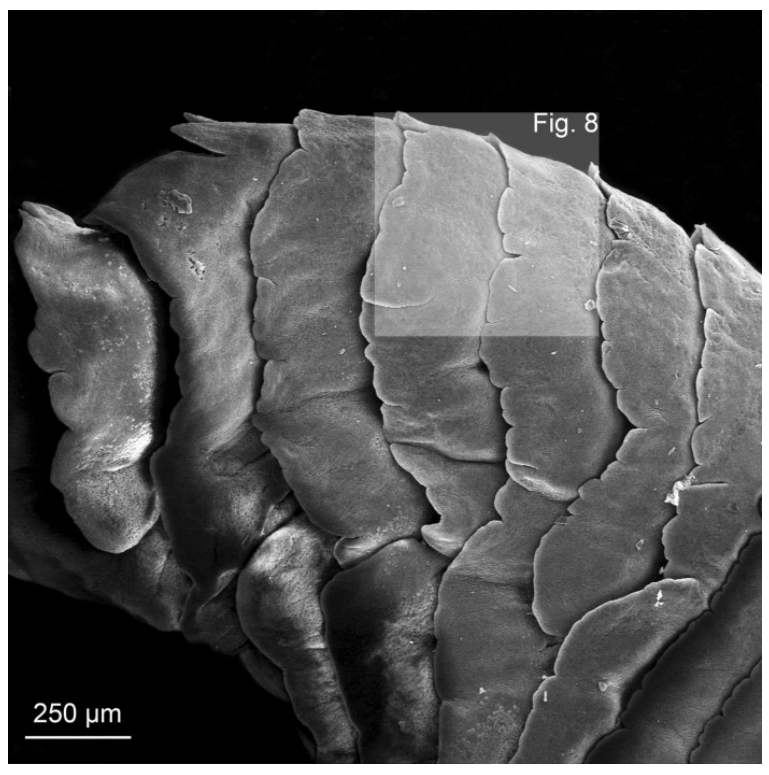

Figure 7. Lingual radix. Papillary ridges.

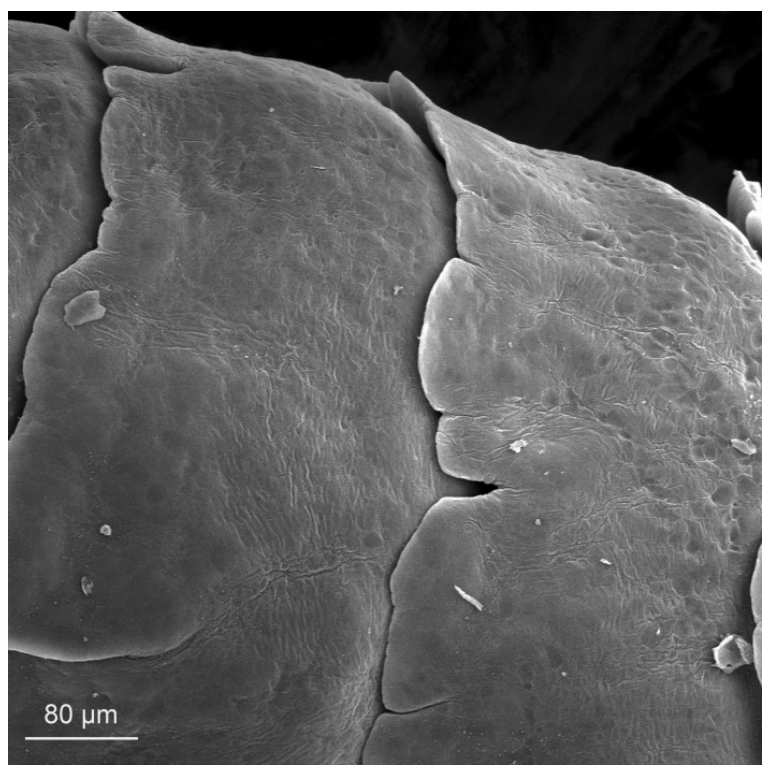

Figure 8. Lingual radix. Detail.

proper connective tissue and numerous striated muscle fibres that originate from the underlying proper lingual muscle (Figs. 9, 10). This muscle is arranged in well-defined fascicles of the horizontal, longitudinal, as well as transversal course.

Light microscopy also reveals the presence of melanin throughout the loose connective tissue of the tongue. The melanin granules are particularly abundant in the papillary cores. No serous or mucous lingual glands, as well as sensory structures were found. No sensory papillae with taste buds were found so all described papillae were considered to be mechanical. 


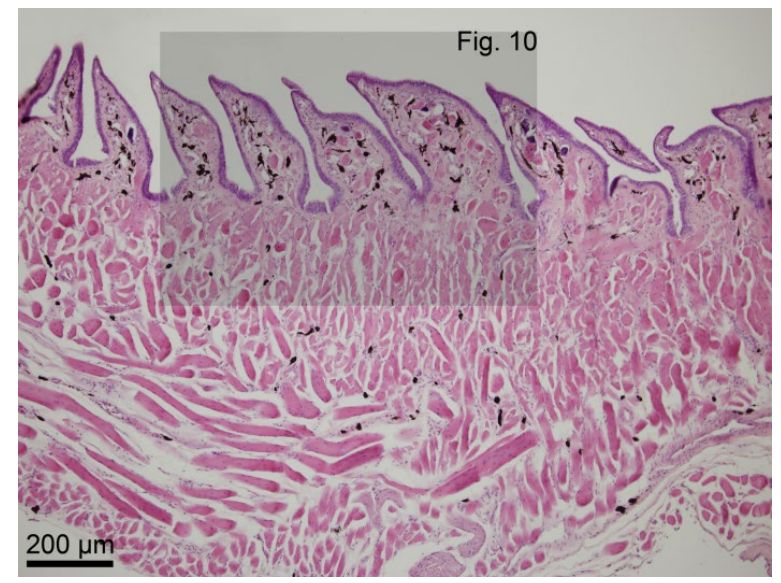

Figure 9. Lingual corpus. Caudally bent papillae containing striated muscle fibres.

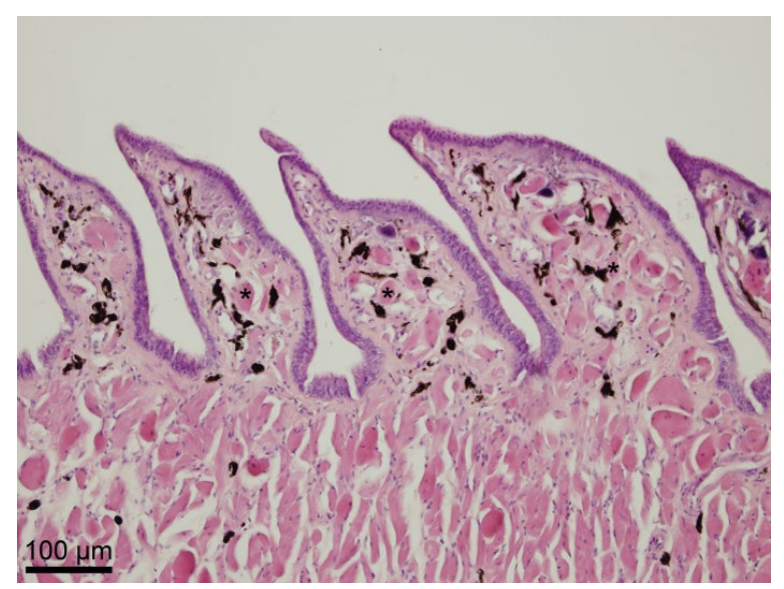

Figure 10. Lingual corpus. Detail. Asterisks indicate striated muscle fibres in the papillary cores.

\section{DISCUSSION}

The tongue of the sand lizard resembles the tongue of the Podarcis sicula in its shape, as well as distribution and type of the lingual papillae [3]. Both these saurian animals, belonging to the Family of Lacertidae, are largely insectivorous [7] and thus this similarity reflects their feeding habits. The observation of the tongue of the $L$. agilis revealed that the organ is divided into three different areas, i.e. apex, body and root. Its lingual apex is bifurcated, as has been described in the Iguana iguana [1, 6], Podarcis sicula [3], Takydromus tachydromoides [13], Scincella tsinlingensis [20] and Pogona vitticeps [21]. Thus, shaping of the lingual apex can be related to an easier access to the vomeronasal organ.

The smooth dorsal surface of the tip of the tongue has also been described in Anolis carolinensis [18],
Tiliqua scincoides [2], Takydromus tachydromoides [13], Pogona vitticeps [21], Podarcis sicula [3], Laudakia stellio [16] and Iguana iguana $[1,6]$. The reason of such an appearance of the lingual apex remains unknown among the researchers so requires further investigation.

Described scale-like papillae on the surface of the lingual body were similar to those found in Takydromus tachydromoides, Lacertidae [13], Scincella tsinlingensis, Scincidae [20] and Gekko japonicus, Gekkonidae [11]. All of these species, as well as L. agilis, are mainly insectivores so such shaping of the papillae possibly helps the food intake and its processing.

It follows from the above mentioned comparison of species that the microscopic structure of the tongue in lizards is not a characteristic feature of the specific families. A more important factor is the type of diet or the way of capturing prey.

Skeletal muscle fibres in the cores of the papillae were also described in Anolis carolinensis. Some authors suspect the papillae can be moved at will [18], although it is hard to imagine moving individual lingual papillae without a relationship to the remaining musculature of the particular area of the tongue.

The epithelium of the dorsal lingual surface is nonkeratinised stratified squamous, as was reported in Anolis carolinensis [18], Scincella tsinlingensis [20], Pogona vitticeps [21], Tarentola annularis [9], Podarcis sicula [3], Iguana iguana [6], Laudakia stellio [16] and Gekko japonicus [11]. This suggests that the occurrence of this type of the lingual epithelium is typical in lizards. A single layer of columnar epithelial cells was reported in Gekko japonicus [11]. Nevertheless, increasing numbers of epithelial layers in the rostrocaudal direction has not been described yet.

Lingual glands were identified in some species of lizards such as Pogona vitticeps [21], Laudakia stellio [16] and Scincella tsinlingensis [20]. Similarly, as in L. agilis, no glands were described in Iguana iguana [6]. It is stated that the salivary glands are located within different structures bordering the oral cavity. The presence of melanin throughout the tongue was also described in Tiliqua scincoides [2].

No taste buds were found on the tongue of the sand lizard. Nevertheless, the taste buds in reptiles are often located in different parts of the oral cavity. In Podarcis sicula, no sensory structures were found on the dorsal lingual surface; however, numerous taste buds were present on the gingival epithelium [3]. 
No taste buds were found also on the lingual surface in Tiliqua scincoides [2]. In the Malayan box turtle (Cuora amboinensis), the taste buds were distributed predominantly on the rostral (prechoanal) part of the palate [10]. In the two-coloured blind snake (Leptotyphlops bicolor) and Texas blind snake (Leptotyphlops dulcis), the taste buds were located only in the mucosa in the vicinity of the vomeronasal organ [5]. On the contrary, the presence of the taste buds in the conical filiform papillae was reported in Tarentola annularis [9]. In the Japanese four-lined ratsnake (Elaphe quadrivirgata), the individual taste buds were located on the sensory papillae, one of which always contained just a single taste bud [17]. It can be assumed that the taste buds in the sand lizard may be located in the other areas of the oral cavity, as was described in the above mentioned reptiles.

Understanding the morphology of the tongue of the sand lizard completes the knowledge of the oral structures in different reptile species. It could also help to explain how different lizards capture the prey.

\section{CONCLUSIONS}

The tongue of the sand lizard is divided into bifurcated apex, corpus and bifurcated radix. Except of the tip of the lingual apex, the dorsal surface of the tongue bears papillae. The lingual epithelium changes from the simple squamous into stratified squamous in the caudal direction. No salivary glands or sensory structures were recognised. This description is to be used mainly for comparative studies. It could also help to understand how different lizards capture the pray.

\section{REFERENCES}

1. Abbate F, Latella G, Montalbano G, et al. Scanning electron microscopical study of the lingual epithelium of green iguana (Iguana iguana). Anat Histol Embryol. 2008; 37(4): 314-316, doi: 10.1111/j.1439-0264.2008.00847.x, indexed in Pubmed: 18279492.

2. Abbate F, Latella G, Montalbano G, et al. The lingual dorsal surface of the blue-tongue skink (Tiliqua scincoides). Anat Histol Embryol. 2009; 38(5): 348-350, doi: 10.1111/j.14390264.2009.00952.x, indexed in Pubmed: 19769569.

3. Abbate F, Guerrera MC, Montalbano G, et al. Morphology of the lingual dorsal surface and oral taste buds in Italian lizard (Podarcis sicula). Anat Histol Embryol. 2010; 39(2): 167-171, doi: 10.1111/j.1439-0264.2010.00992.x, indexed in Pubmed: 20377553.

4. Akbari G, Babaei M, Hassanzadeh B. Morphological study of the European hedgehog (Erinaceus europaeus) tongue by SEM and LM. Anat Sci Int. 2018; 93(2): 207-217, doi: 10.1007/ s12565-017-0391-0, indexed in Pubmed: 28160234.

5. Berkhoudt $H$, Wilson $P$, Young $B$. Taste buds in the palatal mucosa of snakes. Afr Zool. 2015; 36(2): 185-188, doi: 10.1080/15627020.2001.11657136.
6. Cizek P, Krejcirova L, Kocianova I, et al. Light and scanning electron microscopy of the developing lingual papillae in the green iguana, Iguana iguana. Vet Med. 2011; 56(12): 612-618, doi: 10.17221/4439-vetmed.

7. Corbett KF, Tamarind DL. Conservation of the sand lizard, Lacerta agilis, by habitat management. Brit J Herpetol. 1979; 5: 799-823.

8. Delheusy V, Toubeau G, Bels VL. Tongue structure and function in Oplurus cuvieri (Reptilia: Iguanidae). Anat Rec. 1994; 238(2): 263-276, doi: 10.1002/ar.1092380212, indexed in Pubmed: 8154611.

9. El-Sayyad H, Sabry D, Khalifa S, et al. Studies on Tongue of Reptilian Species Psammophis sibilans, Tarentola annularis and Crocodylus niloticus. Int J Morphol. 2011; 29(4): 11391147, doi: $10.4067 / \mathrm{s} 0717-95022011000400012$.

10. Heiss E, Plenk H, Weisgram J. Microanatomy of the palatal mucosa of the semiaquatic malayan box turtle, Cuora amboinensis, and functional implications. Anat Rec (Hoboken). 2008; 291(7): 876-885, doi: 10.1002/ar.20695, indexed in Pubmed: 18449902.

11. Iwasaki S. Fine structure of the dorsal lingual epithelium of the lizard, Gekko japonicus (Lacertilia, Gekkonidae). Am J Anat. 1990; 187(1): 12-20, doi: 10.1002/aja.1001870103, indexed in Pubmed: 2296907.

12. Iwasaki SI. Fine structure of the dorsal epithelium of the tongue of the freshwater turtle, Geoclemys reevesii (Chelonia, Emydinae). J Morphol. 1992; 211(2): 125-135, doi: 10.1002/ jmor.1052110202.

13. Iwasaki S, Miyata K. Scanning electron microscopy of the lingual dorsal surface of the Japanese lizard, Takydromus tachydromoides. Okajimas Folia Anat Jpn. 1985; 62(1): 15-25, indexed in Pubmed: 4034150.

14. Iwasaki S, Asami T, Asami Y, et al. Fine structure of the dorsal epithelium of the tongue of the Japanese terrapin, Clemmys japonica (Cheloia, Emydinae). Arch Histol Cytol. 1992; 55(3): 295-305, indexed in Pubmed: 1419279.

15. Iwasaki S, Wanichanon C, Asami T. Histological and ultrastructural study of the lingual epithelium of the juvenile Pacific ridley turtle, Lepidochelys olivacea (Chelonia, Cheloniidae). Ann Anat. 1996; 178(3): 243-250, indexed in Pubmed: 8712372.

16. Koca YB, Oğuz EO, Osanç E. Morphology, and muscle- and papilla-volume ratios, of the tongue of Laudakia stellio (Agamidae, Squamata): a histological and stereological study. Zoolog Sci. 2007; 24(9): 899-905, doi: 10.2108/zsj.24.899, indexed in Pubmed: 17960995.

17. Nishida Y, Yoshie S, Fujita T. Oral sensory papillae, chemoand mechano-receptors, in the snake, Elaphe quadrivirgata. A light and electron microscopic study. Arch Histol Cytol. 2000; 63(1): 55-70, doi: 10.1679/aohc.63.55, indexed in Pubmed: 10770589.

18. Rabinowitz T, Tandler B. Papillary morphology of the tongue of the American chameleon: Anolis carolinensis. Anat Rec. 1986; 216(4): 483-489, doi: 10.1002/ar.1092160405, indexed in Pubmed: 3799996.

19. Winokur RM. The buccopharyngeal mucosa of the turtles (testudines). J Morphol. 1988; 196(1): 33-52, doi: 10.1002/ jmor.1051960105, indexed in Pubmed: 3373540.

20. Yang C, Wang L. Histological and morphological observations on tongue of Scincella tsinlingensis (Reptilia, Squamata, Scincidae). Micron. 2016; 80: 24-33, doi: 10.1016/j.micron.2015.09.009, indexed in Pubmed: 26421715.

21. Zghikh LN, Vangysel E, Nonclercq D, et al. Morphology and fibre-type distribution in the tongue of the Pogona vitticeps lizard (Iguania, Agamidae). J Anat. 2014; 225(4): 377-389, doi: 10.1111/joa.12224, indexed in Pubmed: 25109482. 